\title{
Making Access to Astronomical Software More Efficient
}

\section{Preben Grosbøl*i}

ESO, Karl-Schwarzschild Str. 2, D-85748 Garching, Germany

E-mail: pgrosboldeso.org

\section{Douglas Tody}

NRAO, 1003 Lopezville Rd, Socorro NM, USA,

E-mail: dtodyenrao.edu

Access to astronomical data through archives and VO is essential but does not solve all problems. Availability of appropriate software for analyzing the data is often equally important for the efficiency with which a researcher can publish results. A number of legacy systems (e.g. IRAF, MIDAS, Starlink, AIPS, Gipsy), as well as others now coming online are available but have very different user interfaces and may no longer be fully supported. Users may need multiple systems or stand-alone packages to complete the full analysis which introduces significant overhead.

The OPTICON Network on 'Future Astronomical Software Environments' and the USVAO have discussed these issues and have outlined a general architectural concept that solves many of the current problems in accessing software packages. It foresees a layered structure with clear separation of astronomical code and IT infrastructure. By relying on modern IT concepts for messaging and distributed execution, it provides full scalability from desktops to clusters of computers. A generic parameter passing mechanism and common interfaces will offer easy access to a wide range of astronomical software, including legacy packages, through a single scripting language such as Python.

A prototype based upon a proposed standard architecture is being developed as a proof-ofconcept. It will be followed by definition of standard interfaces as well as a reference implementation which can be evaluated by the user community. For the long-term success of such an environment, stable interface specifications and adoption by major astronomical institutions as well as a reasonable level of support for the infrastructure are mandatory. Development and maintenance of astronomical packages would follow an open-source, Internet concept.

Accelerating the Rate of Astronomical Discovery, SpS5

August 11-14, 2009

Rio de Janeiro, Brazil

\footnotetext{
*Speaker.

† on behalf of the OPTICON Network on 'Future Astronomical Software Environments'.
} 


\section{Introduction}

The efficiency and speed with which one can obtain new astronomical results depend upon a long chain of facilities and tools such as telescopes, instruments, data archives, and software packages. Although many discoveries are made using new, more capable telescopes and instruments, one should not underestimate the importance of easy access to both archival data and state-of-theart software. The combination of multi-wavelength data (e.g. obtained through data archives) and application of new algorithms for analysis of data can lead to new understanding of astronomical phenomena.

In this paper, we focus on how access to and sharing of astronomical software can be made easier and thereby increase the speed with which new results are obtained. The prime concern is the efficiency of turning raw data into astronomically relevant information. First an overview of the current situation is given, followed by considerations on possible ways to improve it. An architectural concept for a future astronomical software environment is then presented as discussed by OPTICON and the USVAO (formerly NVO). Finally, steps needed to provide the astronomical community with an efficient software environment for future data challenges are reviewed.

\section{Current state of affairs}

Removal of the instrument signature from and calibration of raw data are often done by applying standard pipelines offered by the provider of the facility. Although they frequently are based upon familiar legacy systems (e.g. IRAF, MIDAS, CPL/ESO, Starlink, AIPS), users may find it difficult to disentangle their internal structure, and be forced to treat them as 'black boxes'. This limits the ability of a researcher to optimize a pipeline for a particular data set by either changing its default parameters or by fully replacing some of its modules. While a generic pipeline will provide standardized processing for a wide variety of raw data sets, it will never give the best results for a specific data set. Customized processing by the researcher is often necessary to produce new discoveries.

Reduced, calibrated data are typically obtained through dedicate pipeline processing or directly from archive data using Virtual Observatory (VO) tools. In either case, a set of analysis tools needs to be applied in order to extract the astronomically interesting information. Such tools are still mainly available through legacy systems although many of them are maintained only at a marginal level and new developments are currently quite limited. Most of these legacy systems were developed to satisfy a certain user community (e.g. with data originating from special detectors or instruments, often for wavelength specific regimes). People analyzing multi-wavelength data or having special requirements for tools are often forced to use a combination of several systems as well as stand-alone applications. Although this is possible, it adds a significant overhead as several, non-compatible scripting languages and data formats may have to be applied. Differences between systems also make it complicated to compare different application packages for the same purpose and thereby identify the optimal one for a given set of data.

The current scenario has several disadvantages due to the fragmentation of efforts. Each system has to expend effort to follow the general Information Technology (IT) development such as support of new operating systems or standards. This is costly and may lead to legacy systems 
being unavailable on modern platforms. Since the systems have their own internal standards and conventions, it is difficult for an individual user to learn them in detail to be able to fully utilize the facilities provided. Often the user concludes that it is simpler to re-write a task even if it is already available in a system unfamiliar to the user.

\section{Vision for the future}

It is important to recognize the boundary conditions when one considers how to improve the current situation. Large organizations with strict operational requirements as well as large projects with carefully constrained deliverables may demand full control of code used for critical tasks [1]. On the other side of the scale, users would like easy access to all available software but without being forced to use a specific system. This suggests that any new software approach must be based upon a modular environment integrating software from many sources and must depart from the old monolithic, vertically structured model. The only solution is to develop cross-cutting standards to integrate software from different projects into a common environment. The primary drivers for establishing a new common environment [2] for astronomical software are:

- Efficient access: A simple, shared interface specification for astronomical code would significantly ease sharing and comparison of available code and packages. Applications conforming to such specifications could be directly included within and accessed from a common environment.

- Separation of astronomy and IT: A clear distinction between astronomical code and IT infrastructure would make it much simpler to upgrade or exchange code in the two areas at different timescales. It would also make it easier to adopt new IT technology as it becomes available and thereby reduce the development and maintenance burden.

- Scalability: With the exponential increase in data volumes presently occurring within astronomy, it is becoming increasingly essential to develop new algorithms and procedures on an easily accessible, local computer and then migrate the software to distributed cluster systems for a final processing of large data sets. Provision of scalability at the framework level, making large- and small-scale processing systems comparable is critical to making this possible.

- Customized implementations: A single system implementation is seldom adequate to encompass the full range of use-cases from small end-user laptops to large computational clusters integrating entire observatory data systems. A layered, modular concept would allow different organizations to provide their own incarnations of the environment, all conforming to the common interface specifications, optimized to suit their specific demands while still sharing common astronomical code and applications packages.

By following these key points, one could establish a body of astronomical code shared and maintained by the community in the spirit of open-source, Internet developments. The common interface specifications would ensure easy access to packages while not forcing anybody to use a specific implementation of the IT infrastructure. This would secure the investment in astronomical algorithms even in a changing IT world. The needs of observatory data processing would 


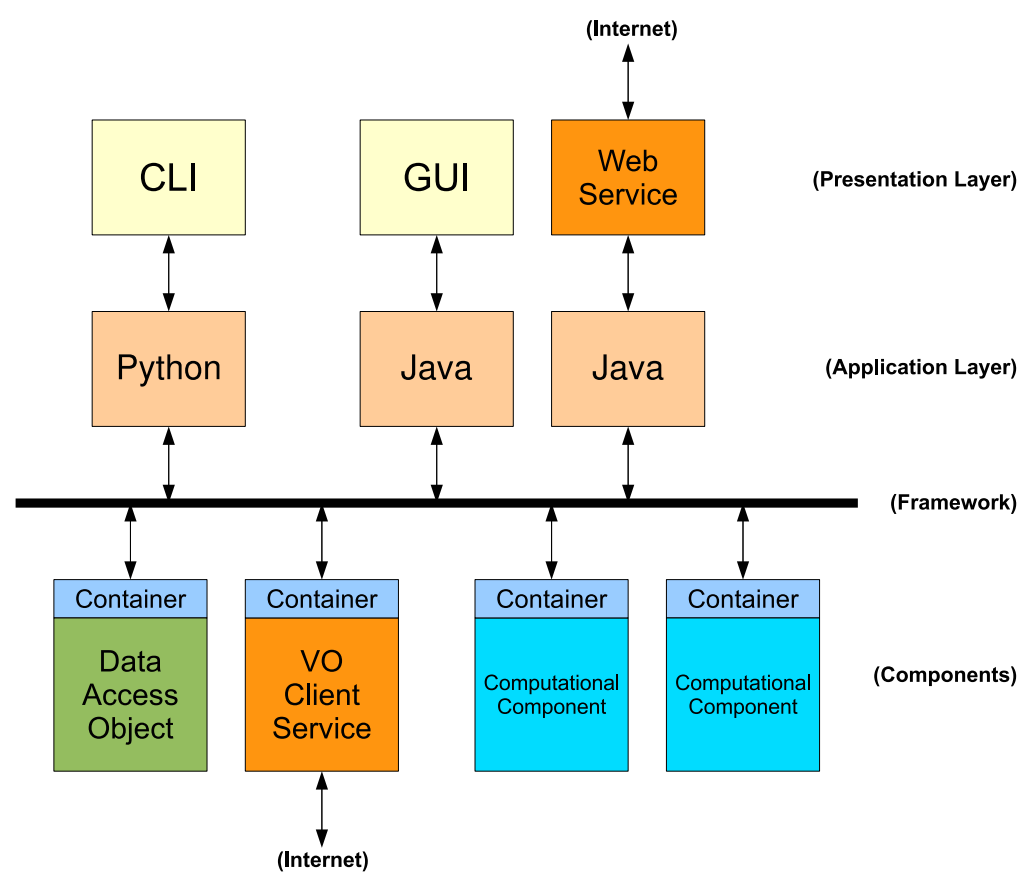

Figure 1: Architectural concept for common environment.

be addressed while at the same time providing integration with virtual-observatory standards for distributed multi-wavelength data analysis capabilities.

\section{Architectural concept}

The OPTICON network on 'Future Astronomical Software Environments' was started in 2004 to discuss requirements, architectural concepts and high-level design of an environment which would be able to satisfy future needs of astronomical users for processing and analysis of their data. The Network included participants from US and Europe representing both end-users and people associated to major data systems. First, main use-cases were developed such as pipeline processing, desktop analysis of data, and heavy computational tasks using clusters of distributed computers. With these scenarios in mind, high-level requirements [3] for future environments were established and reviewed by the community via the Internet as well as face-to-face meetings.

Considering both use-cases and requirements, an architectural concept [ [] for future environments was outlined as shown in Fig. 1. It is based upon a layered structure (commonly used in the IT world) and provides support for scalability from laptops to large, distributed computer systems. The upper layer (presentation) offers different options for user interfaces which could range from simple command line input (CLI) to advanced graphical ones (GUI). It would also be possible to provide an interface to other software tools (e.g. browsers) and thereby establish Web services. The next layer (application) provides for high-level astronomical applications written in scripting languages like Python, Ruby or a Unix shell. In special cases, one could also use other languages such as Java which offers easy access to Web, VO, and GUI packages. Typical applications at this layer 
manage computational tasks and the results of such tasks, but not the computations themselves. The importance for this layer is flexibility and easy change rather than optimal performance.

The framework layer connects the application layer scripts with high performance, computational code at the component layer using a flexible, distributed communication framework. This provides a high level of scalability since computational tasks can be executed either on a local host or at any available system in the network, fully transparent to the upper layers. Asynchronous execution could also be supported allowing users to perform heavy computational tasks (e.g. pipeline processing) as background processes. The framework would be based upon a software-bus or messaging system, as is commonly used for distributed processing. By selecting one of the numerous free implementations (e.g. VO SAMP, D-bus or openMPI), a major part of the framework would be readily available. Two other parts would need some astronomical customization, namely: a) parameter passing mechanism which would transfer parameters and results between application scripts and computational components, and b) package management being responsible for the definition and installation of new astronomical packages into the environment.

The main computational code is placed at the lower layer (organized as software modules known as 'components') which link to the framework through a standardized interface called the 'container'. On one side a container connects to the framework while on the other side it provides a stable, common interface to the astronomical code. This isolates the astronomical code from the IT infrastructure, represented by the framework, which often changes faster then astronomical algorithms. Separate containers for different coding languages (e.g. C/C++, FORTRAN, Java) are required but special binding libraries to ease support for legacy systems are possible. Different types of components could be envisioned such as simple computational tasks or stateful services which would allow complex interaction with the user through GUI's. Special components to access data or Web services (e.g. provided by VO) could also be made.

This architectural concept has many advantages compared with monolithic systems and independent developments. The clear separation between astronomical code and IT infrastructure makes it easy to obtain the latter through public-domain IT projects and thereby reduce maintenance cost. Further, the astronomical parts can be migrated to new IT infrastructures with a minimum of labor. Organizations that demand full control can implement their own environment but still benefit from algorithms using the common interface specifications.

A common, accepted interface to the environment would simplify the integration of new astronomical code. Individual astronomical developers would have direct access to the environment and its general facilities by using the standardized interface. Their code would also be readily available to the community through a simple packaging and package distribution mechanism.

\section{Way forward}

With general agreement on this architectural concept [5], the OPTICON network with the USVAO have started a detailed design study to outline the main parts of the environment and their interfaces. The next step is a prototype implementation of the most important parts (i.e. parameter passing, package management, messaging and execution) as a proof-of-concept. This will lead to a refinement of interface specifications and structure. Finally, a minimal reference implementation for a desktop system is foreseen to provide a test-bed which can be evaluated by the community. 
It would be available initially for at least the Linux and MacOSX operating systems with support for Python for scripting, Java for VO and Web access, and C/C++/FORTRAN for compiled code. Parallel to this effort, current legacy systems will be contacted to evaluate the feasibility of adapting their most important packages to the new environment.

Making a basic version of the environment available to astronomers will not convince them that it is worthwhile using it. This can only be done by making it easy to install and handle on common desktop systems. The version must provide access to important legacy applications and allow new software to be added in a trivial manner.

The long-term success of the concept proposed will depend on other, more administrative factors. The common interface specifications must be stable which require them to be controlled by an international body (e.g. an IAU working group as for the FITS standard, or an appropriate subgroup operating within the IAU or IVOA frameworks). Basic support and maintenance of a reference environment have to be secured even if it only provided a simple desktop version.

\section{Conclusions}

The current situation with a multitude of largely incompatible legacy or more modern but narrowly focused systems for data processing and analysis is not satisfactory since it makes access to software packages more difficult and requires significant resources for maintenance. To remedy these problems, a new approach is required based upon the concept of a common astronomical software framework which allows flexible integration of both new and legacy code, which is scalable to leverage modern computational hardware architectures while meeting the challenge of exponentially increasing data volumes, and which separates astronomical code from the IT infrastructure to provide stability for critical astronomical code while allowing rapid uptake of new IT technologies.

The requirements and architectural concept for such an environment have been discussed within an OPTICON Network with US and EU participation. It is proposed to adopt a modular, layered model based upon a framework supporting messaging and execution in a distributed system. Major parts of the framework can be taken directly from general open-source IT projects while other areas, like parameter passing, need to be customized for astronomical applications. The isolation of IT infrastructure will safeguard the astronomical code against the rapid changes in the IT world. The generic, modular structure would allow easy integration of both new and legacy packages. This would significantly increase the efficiency with which astronomers can access a wide variety of available astronomical software. An efficient access to software tools would directly lead to accelerating the rate of astronomical discovery.

\section{Acknowledgements}

This paper is based on discussions in the OPTICON Network 3.6 on 'Future Astronomical Software Environments'. We especially want to thank INAF for its support of this work. The project has been supported by OPTICON funded by the European Commission under Contract no. RII3-CT-2004-00001566 and the USNVO under NSF Cooperative Agreement AST0122449. 


\section{References}

[1] Grosbøl, P., Banse, K., Ballester,P. 1999, Pipeline Processing of Data in the VLT Era in ADASS VIII, Eds. D.M.Mehringer, R.L. Plante and D.A. Roberts, ASP Conf. Ser. 172, 151

[2] Grosbøl, P., Ballester, P., Banse, K. 2004, A Common Data Analysis Environment: Do we need one? in Towards and International Virtual Observatory Eds. P.J.Quinn and K.M.Gorski, ESO Astrophysics Symposia, (Springer, Berlin), 281

[3] Grosbøl, P., Banse, K., Tody, D., Cotton, W., Cornwell, T., Ponz, D. et al. 2005: Requirements for a Future Astronomical Data Analysis Environment in Astronomical Data Analysis Software and Systems XIV, Eds. P.L.Shopbell, M.C.Britton and R.Ebert, ASP Conf. Ser. 347, 124

[4] Tody, D., Grosbøl, P., Garilli, B. et al. 2006, An Open Architecture and Framework for Astronomical Data Processing and Analysis in Astronomical Data Analysis Software and Systems XV, Eds. C.Gabriel, C.Arviset, D.Ponz, E.Salano, ASP Conf. Ser. 351, 331

[5] Tody, D., Grosbøl, P. 2007, Astronomical Data Processing and VO in Astronomical Data Analysis Software and Systems XVI, Eds. R.A.Shaw, F. Hill and D.J. Bell, ASP Conf. Ser. 376, 681 